\title{
Sea clutter constituent synthesis approach based on a new decomposition model
}

\author{
Shengmiao Zhang*, Jun Li, Zishu He, Wei Zhang and Huiyong Li
}

\begin{abstract}
In this paper, a sea clutter decomposition model is newlxy proposed. The decomposition structure is organized according to a comparison study between measured sea clutter and Lorenz chaotic signals. Based on the decomposition model, a sea clutter constituent synthesis approach is developed to reconstruct sea clutter series with neural networks. Simulation results demonstrate the effectiveness and stability of the proposed approach.
\end{abstract}

Keywords: Sea clutter; Decomposition model; Chaotic modeling

\section{Introduction}

Sea clutter modeling is a significant issue in radar signal processing. Generally, a proper sea clutter model is important in radar signal processing for three reasons. Firstly, it can describe the underlying dynamics of sea clutter. Secondly, with a proper model, effective detectors can be developed for target tracking in sea clutter. Finally, it is also useful for generating a representative clutter signal for radar system's testing and receiver algorithm's development [1].

Various models for sea clutter have been proposed with simulation techniques [2]. Traditionally, sea clutter is commonly described as a non-Gaussian stochastic process [3], such as Weibull, log-normal, $\mathrm{K}$, and compound Gaussian distributions [4-8]. A major drawback of statistical models is that they provide very little information about the underlying dynamics of sea clutter, which might be useful for improving signal processing performance [9-11]. Moreover, statistical models are used to describe a large amount of data's distribution characteristics. In practical applications, a coherent processing interval (CPI) is always limited (such as 64 or 128 pulses). It can be inappropriate to refer to this small amount of data with statistical models.

While statistical models still have their problems, and the nonlinear behavior within sea clutter has been confirmed [1], fractal model and chaotic model of sea clutter are proposed as innovative methods. By applying fractal analyses techniques (such as the Hurst parameter estimation), the

\footnotetext{
* Correspondence: miaomiao871@sina.com

School of Electronic Engineering, University of Electronic Science and Technology of China, Chengdu, China
}

fractal modeling of sea clutter has been shown to be effective in target detection [12-14]. At the mean time, chaotic modeling of sea clutter is also studied. In the past decades, several researchers have reported that sea clutter is more likely to be chaotic rather than purely random [15-17]. As a result, a lot of effort has been made to fit sea clutter with chaotic models [18-20]. However, the fitting performance of these reconstruction methods is not good enough. As a consequence, the chaotic nature of sea clutter has been reexamined. It has been suggested that sea clutter is not the result of deterministic chaos, but it might be a stochastic chaotic process [1]. This conclusion indicates that sea clutter might be "partially chaotic". Due to the fact that chaotic processes have been realized with analog circuits, chaotic modeling approach of sea clutter can be utilized not only in sea clutter underlying dynamic analysis and target detection but also in clutter generation applications, which makes the chaotic modeling of sea clutter still worthy of study.

This article focuses on improving sea clutter's chaotic modeling performance. As an intelligent computation approach, neural networks are widely employed in sea clutter's chaotic modeling for their ability to approximate the unknown functional form of a chaotic system. By using neural networks as one-step predictors, targets within sea clutter background can be detected from the prediction error. One of the commonly used neural networks is the radial basis function (RBF) network [21-25], which has been utilized for sea clutter modeling and target detection in the last two decades [26-28]. In reference [18], an RBF neural network is used to perform a non-recursive single-point prediction of the real measured IPIX radar sea 
clutter data. Unfortunately, when this approach is used to model a spiky sea clutter, the predictable samples' number is small and the overall fitting performance is unstable. Advanced machine learning techniques are used to deal with this problem, such as multi-model RBF approach (MM-RBF) and genetic algorithm multi-model RBF approach (GA-MM-RBF) $[9,20]$. Both approaches share the same assumption that sea clutter can be divided into different segments with different chaotic dynamic models in a time domain. In this work, in order to give a better understanding of our newly proposed approach, we refer to the MM-RBF approach as a "time division" approach. Note that the time division approach adjusts the intelligent predictors' structure to deal with the spiky sea clutter without any preprocessing of the sea clutter itself.

In this paper, a sea clutter decomposition model (SCD model) is proposed via decomposing sea clutter in the frequency domain. The SCD model is motivated by the observation of a comparison study between real measured sea clutter time series under different sea states and typical chaotic signals. The comparison study is necessary since the goal of our work is to fit sea clutter with chaotic models. The comparison results suggest that 1 ) the original sea clutter series are quite different from chaos signals in waveforms and spectrums, and 2) with a two-step decomposition process, a chaotic likely component in sea clutter is found to have similar characteristics with chaotic signals in both the waveforms and spectrums. In fact, no matter what kind of nonlinear predictor is utilized, a right object to fit is crucial for a good fitting performance. The comparison results indicate that instead of regarding the whole original sea clutter as chaotic series, the chaotic likely component in sea clutter is probably a more appropriate fitting object to be approximated with chaotic models.

Based on the proposed SCD model, sea clutter is modeled by a constituent synthesis approach. We refer to this approach as a sea clutter constituent synthesis (SCCS) approach for short. In the SCCS approach, as an inverse process of the SCD model, sea clutter is regarded as a compound signal of two major components: a relatively low-frequency envelope likely signal, and a relatively highfrequency "de-enveloped" signal, where the "de-enveloped" signal is the "chaotic likely component" (note that these two components are different from the "texture" and "speckle" components in compound Gaussian distribution model). In this work, chaotic modeling of sea clutter is realized by fitting these two components with RBF predictors separately.

Simulation results with measured sea clutter confirm that the SCCS approach can achieve a good and stable fitting performance. It is worth noting that the simulation results shown in this work are used to illustrate that the proposed SCD model can be used as a preprocessing method. The fitting performance might be improved when other advanced predictors (such as the Support Vector Machine (SVM) model [29] and the mentioned GA-MM-RBF model) are involved instead of the very basic RBF model used in this paper.

The proposed SCD model and SCCS approach have the flowing advantages: 1) theoretically, by using the right component as the chaotic modeling object, the SCCS approach is more compliant with the original motivation of the sea clutter's chaotic modeling; 2) in practice, the utilization of SCD model leads to a robust performance of the SCCS approach in different kinds of spiky sea clutter situations; 3 ) from the development perspective, the SCD model provides a new angel to analysis and modeling sea clutter.

This paper is organized as follows: a brief description of sea clutter chaotic modeling with neural networks is presented in Section 2. Section 3 introduces the newly proposed sea clutter decomposition model as well as the constituent synthesis approach for sea clutter reconstruction. In Section 4, performance of the proposed approach is analyzed using real measured IPIX radar data. Finally, the work is summarized and concluded in Section 5.

\section{Sea clutter's neural network prediction approach}

Neural networks use a training algorithm to adjust the weights and thresholds in order to optimize the universal function. This adjusting process is known as a training or learning process. After the training process, the optimized universal function can be used to approximate a predictive process (also called as a target process). Mathematically, the prediction computation process (also called as a testing process) in a trained neural network is described by the following equation [9]:

$$
x[n]=f(x[n-1], x[n-2], \cdots, x[n-N])
$$

where $(x[n-1], x[n-2], \cdots, x[n-N])$ is an $N$ sample input time series; $x[n]$ is the predicted value in time step $n$, and $f$ is a nonlinear one-step prediction function which is determined during the training process.

When a set of sea clutter data is used in the training process, after an effective training process, the trained neural network function $f$ will be able to approximate the underlying nonlinear function in this set of sea clutter data. In target detection applications, the trained network is used as a one-step predictor. The presence of target within sea clutter will be detected if the prediction error is abnormal. So the detection performance is determined by the prediction accuracy.

A radial basis function (RBF) network is used to predict real sea clutter data collected by the McMaster IPIX Radar system at the east coast of Canada, from a cliff top near Dartmouth, Nova Scotia (http://soma.ece.mcmaster.ca/ ipix/). The IPIX radar is an X-band land-based radar and 
the IPIX radar data sets are widely used in research works about sea clutter issues. Five sets of measured target-free range bin staring data are used in this paper. Some characteristics of these datasets are shown in Table 1, where the "sea state code" and "characteristics" come from the world meteorological organization sea state code. Since each dataset has 131,072 samples, only a segment of them is employed for training and testing process in each experiment.

In this section, data $S 1$ is utilized in simulation experiments. The number of training samples has complex influence on the prediction performance of the trained network. Since this issue is not the focus of this work, an appropriate samples' number is chosen to obtain general results. In each experiment, 5000 samples from $S 1$ are invoked as training data. The RBF network is constructed with MATLAB library functions from the neural network toolbox without any adjustment of the center of each neuron. After the training process, the next 2000 samples are used to test the prediction performance of the trained network. The predictor works in a one-step fashion, which means that the most recently acquired real measured samples are used as the network input for the next prediction step. The start points of the training samples are chosen randomly and different in each experiment, as shown in Fig. 1. Although the training and testing data series of each experiment are intercepted from the same data set, their characteristics can be quite different since they have different start points.

Two typical prediction results are presented in Figs. 2 and 3. The corresponding measured data series is shown for comparison. The training samples' start point of the results shown in Fig. 2 is data point 5000, while the start point of the experiment illustrated in Fig. 3 is data point 1.

To evaluate the performance of a chaotic modeling approach of sea clutter, prediction errors and predictable samples' number are two commonly used indicators [18]. In Fig. 2, with the increase of the testing samples' number, prediction errors are tended to increase. In fact, the prediction performance shown in Fig. 2 is rarely good in most times of experiments. In most of the other experiments (with different training samples' start points), the predicted data are unable to fit the measured data and become divergent, which means that only a very short segment of testing samples is predictable. One typical result is illustrated in
Fig. 3. Simulation results in this section indicate that using a single-RBF network to fit sea clutter directly is not effective enough due to its instability.

\section{Sea clutter comparative analysis, a decomposition model, and a reconstruction approach}

\subsection{A comparative study}

Since the original motivation and potential to describe sea clutter with chaotic model come from the observation that sea clutter displays qualitative similarities to chaotic signals, a comparative study between real-life sea clutter and chaotic signals generated by the well-known Lorenz chaotic system is developed, which examines the similarity between measured sea clutter and chaotic signals in an intuitive way.

The Lorenz chaotic system is given by [30]

$$
\left\{\begin{array}{l}
\dot{x}=-\sigma x+\sigma y \\
\dot{y}=r x-y-x z \\
\dot{z}=x y-b z
\end{array}\right.
$$

where $x, y$, and $z$ are state variables and $\sigma, r$, and $b$ are Lorenz parameters. The system is chaotic when all the Lorenz parameters are greater than zero and satisfy the flowing constraints:

$$
\left\{\begin{array}{l}
\sigma>b+1 \\
r>\frac{\sigma(\sigma+b+3)}{\sigma-b-1}
\end{array}\right.
$$

The top subplot in Fig. 4 shows a typical 2000-point waveform of state $y$ obtained via numerical integration of the dynamical system given by Eq. (2) with $\sigma=267, r=595$ and $b=100$. The bottom subplot shows a typical waveform from the IPIX radar measured dataset S1. Their corresponding spectrums are shown in Fig. 5. Spectrums are calculated by FFT (the DC component has been removed), and the FFT results are normalized to a range of $0-1$ for the ease of comparison.

In Fig. 4, the amplitude of the Lorenz signal has a stable boundary, while the fluctuation of the amplitude of the sea clutter is fairly obvious. In Fig. 5, the Lorenz series has a broad flat spectrum, while the sea clutter series has a broad flat spectrum with a high-energy component in the very low-frequency region. Note that this low-frequency

\begin{tabular}{|c|c|c|c|c|c|}
\hline Datasets & S1(Hi-State) & S2(Lo-State) & S3(\#19) & S4(\#31) & S5(\#280) \\
\hline Range (m) & $900-1095$ & $1200-1410$ & $5475-5670$ & $2574-2769$ & $2550-2745$ \\
\hline $\operatorname{RPF}(\mathrm{Hz})$ & 1000 & 2000 & 1000 & 1000 & 1000 \\
\hline Wave height (m) & $1.8(\max .2 .9)$ & $0.8(\max .1 .3)$ & 2.0 & 0.9 & 1.4 \\
\hline Sea state code & $4-5$ & $3-4$ & 4 & 3 & 4 \\
\hline Characteristics & Moderate/rough & Slight/moderate & Moderate & Slight & Moderate \\
\hline
\end{tabular}

Table 1 Characteristics of IPIX radar measured datasets used in this work 


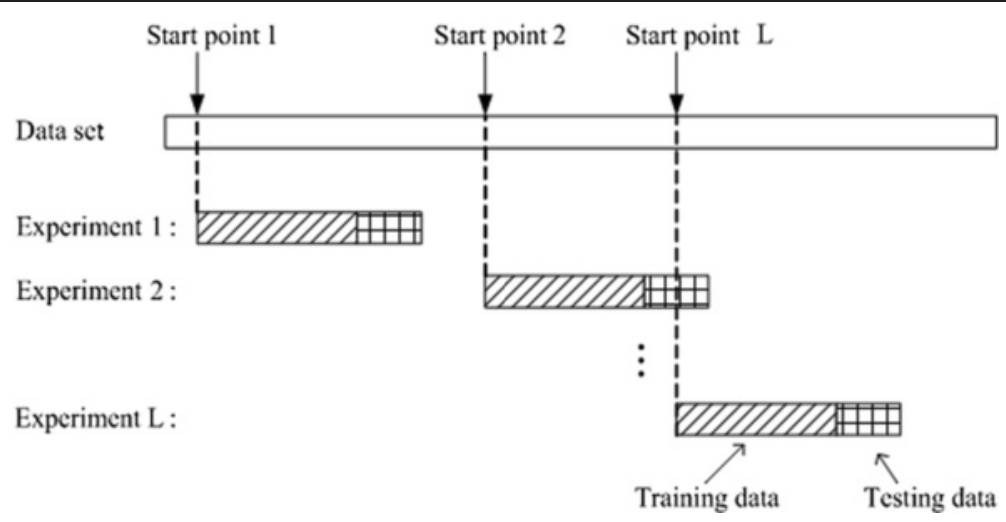

Fig. 1 Data interception schematic. Start points of the training samples are chose randomly and different in each experiment

component is caused by the fluctuation of the sea clutter's spiky envelope.

As mentioned earlier, the potential to model sea clutter using neural networks comes from not only the fact that neural networks can be used to reconstruct nonlinear systems such as chaotic systems but also the observation that sea clutter display qualitative similarities to chaotic signals. Considering the fact that sea clutter as a whole signal is not similar enough to chaotic signals, it is reasonable to suggest that a decomposition process is needed to find an appropriate component in sea clutter, which is similar to chaotic signals and can be well reconstructed by neural networks.

\subsection{The sea clutter decomposition model}

The proposed sea clutter decomposition (SCD) process and the example waveforms at each step of SCD process are shown in Fig. 6. The measured data $S 1$ is utilized to construct example waveforms.

The measured base-band sea clutter signal takes the form of a time series, $\mathbf{c}[n]=\left\{c_{n}, n=1,2, \cdots\right\}$ (waveforms a in Fig. 6). In step $1, \mathbf{c}[n]$ is divided into a relatively lowfrequency curve signal and a high-frequency signal by a low-pass filter. Output signal of the low-pass filter is denoted as

$$
s_{0}[n]=f_{\mathrm{LP}}(\mathrm{c}[n])
$$

With the group delay caused by the low-pass filter taken into account, the low-frequency part (waveforms b in Fig. 6) in $c[\mathrm{n}]$ is

$$
\mathrm{s}[n]=s_{0}[n-k]
$$

where $k$ refers to the discrete group delay. Then the residual signal (waveforms c in Fig. 6) is calculated by

$$
\mathbf{r}[n]=\mathbf{c}[n]-\mathbf{s}[n]
$$

The envelope of the residual signal $\mathbf{r}[n]$ is shown in Fig. 7 with a dotted line. Obviously, the waveform of $\mathbf{r}[n]$ still has certain fluctuation. In order to find a chaotic similar component in the sea clutter signal, $\mathbf{r}[n]$ need be further decomposed.

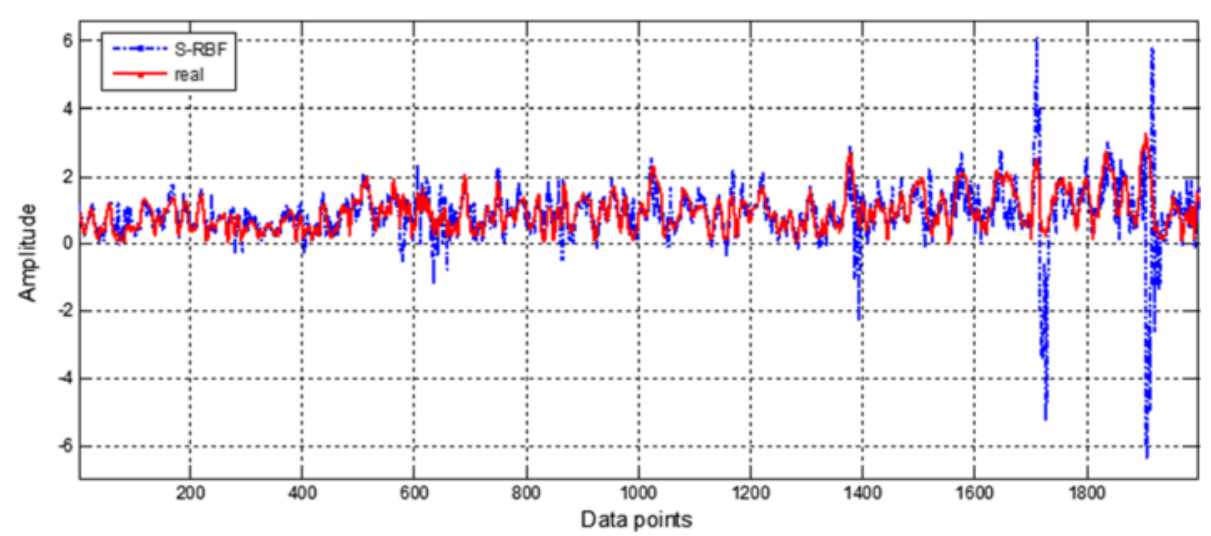

Fig. 2 Prediction results using single-RBF predictor with data start point at 5000th. A rarely good prediction result obtained by single-RBF predictor. Five thousand samples are used for training, and the next 2000 samples are used for testing. Although the predicted series are convergent, with the increasing of the testing samples' number, prediction errors have a tendency to increase 


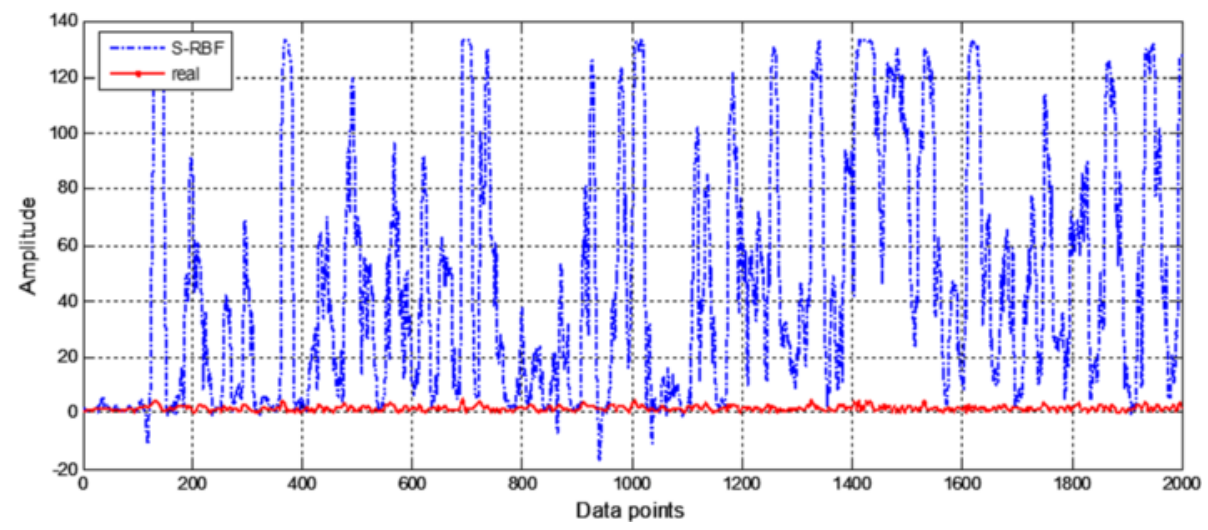

Fig. 3 Prediction results using single-RBF predictor with data start point at 1st. A typical prediction result obtained by single-RBF predictor (5000 samples are used for training and the next 2000 samples are used for testing), in which the predicted data were unable to fit the measured data and became divergence

Unlike the first step, low-pass filter is not used in step 2. Instead, the residual signal is "de-amplitude-modulated (de-AM)" by the low-frequency signal $s[\mathrm{n}]$ obtained in the first step. The reason lies in the interesting observation that the envelope of $\mathbf{r}[n]$ is very close to $s[n]$, as shown in Fig. 7.

The underlying cause of this observation is unknown yet, but we can make use of it to simplify the decomposition process. So the low-frequency signal is employed in step 2 directly. Then $\mathbf{r}[n]$ is "de-AM" by $\mathbf{s}[n]$ and get the de-AM signal $\mathbf{d}[n]$ :

$$
\mathbf{d}[n]=\frac{1}{\mathbf{s}[n]} \odot \mathbf{r}[n]
$$

where $\odot$ refers to the Hadamard product (element-byelement multiplication), and $\mathbf{d}[n]$ is named as a "de-AM signal" (waveforms $d$ in Fig. 6). Waveforms shown in
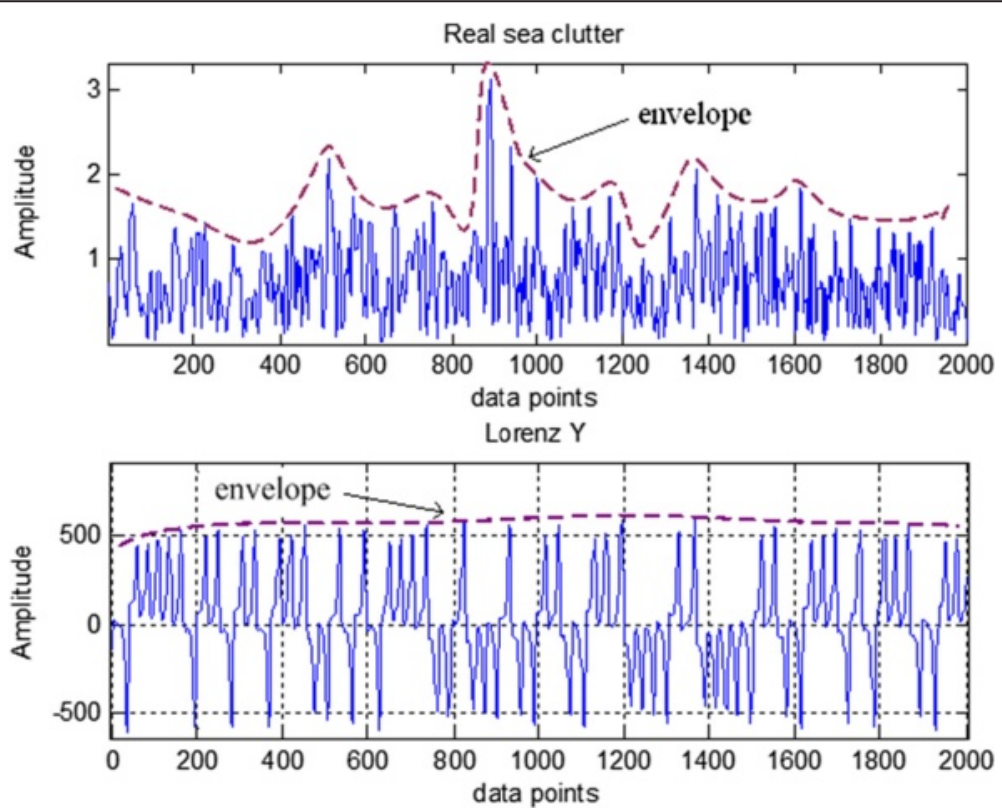

Fig. 4 Waveforms comparison of measured sea clutter and Lorenz signal. The 2000 samples' waveform in top subplot is intercepted from dataset S1, while the 2000 samples' waveform in bottom subplot is a typical waveform of Lorenz signal obtained via numerical integration. Here the parameters of the Lorenz dynamical systems are set as $\sigma=267, r=595$, and $b=100$ 


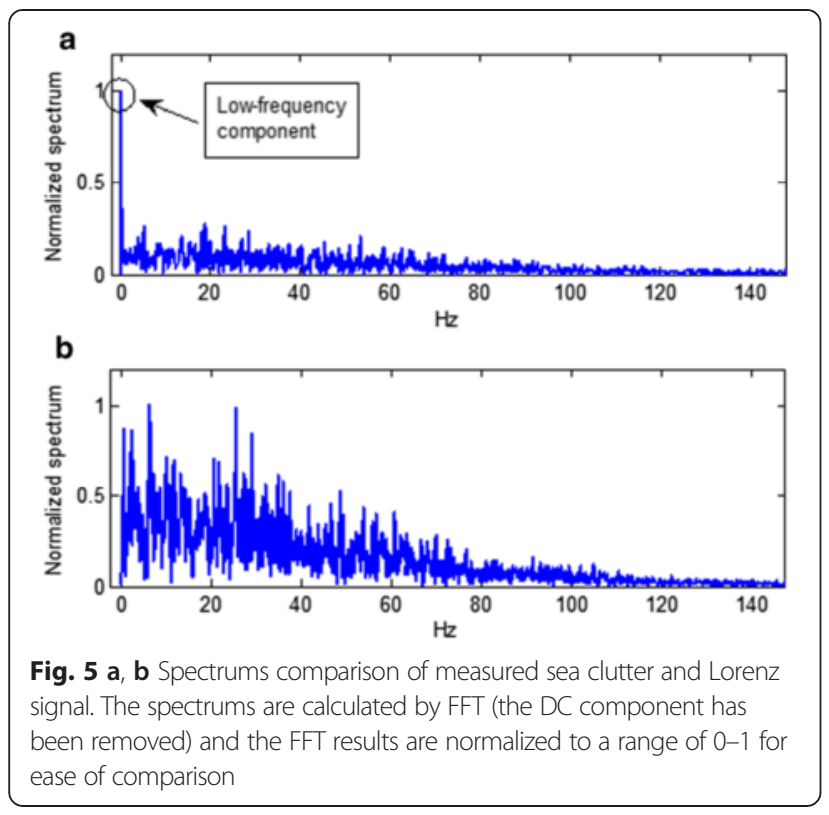

Figs. 6 and 7 have the original length (131,072 samples) of the measured IPIX radar data sets. In order to make a fair comparison, a piece of signal with 2000 samples is intercepted randomly from $\mathbf{d}[n]$ :

$$
\mathbf{d}_{\text {piece }}=\mathbf{d}[p: p+1999]
$$

where $p$ refers to the random data start point. The waveform and spectrum of a typical $\mathbf{d}_{\text {piece }}$ are illustrated in Fig. 8 . Again, the spectrum is calculated by FFT (the DC component has been removed) and the FFT result is normalized to a range of $0-1$ for the ease of comparison.

Comparing Fig. 8 with Figs. 4 and 5, although the bandwidth of spectrums shown in Figs. 5 and 8 are different, the envelope of these spectrums share a similar shape, and both the waveforms and spectrums of the de-AM signal are quite similar to that of chaotic signals. Actually, the bandwidth of a chaotic signal can be adjusted by changing its system parameters, such as $\sigma, r$, and $b$ in the Lorenz system. So the bandwidth difference between sea clutter and a typical piece of chaotic signal will not affect the spectrum similarity between them. Therefore, the de-AM signal $\mathbf{d}[n]$ might be an appropriate component in sea clutter and can be described by a chaotic model.

The two-step decomposition process described by Eq. (4)-(7) is referred to as a sea clutter decomposition model, which can be used to divide sea clutter series and obtain the chaotic likely component. After the proposed SCD process, measured sea clutter series is divided into $\mathbf{s}[n]$ and $\mathbf{d}[n]$. In the next section, an inverse process of SCD process will be used to reconstruct the whole sea clutter.

\subsection{The sea clutter constituent synthesis approach}

In this section, a sea clutter constituent synthesis (SCCS) approach is proposed based on the SCD model. A block diagram of the proposed SCCS approach is shown in Fig. 9. It consists of one decomposition model, two predictors, and one synthesis model. The decomposition model is used to decompose $c[n]$ into $\mathbf{s}[n]$ and $\mathbf{d}[n]$. The predictors are used to approximate the unknown dynamical functions in $\mathbf{s}[n]$ and $\mathbf{d}[n]$, while the synthesis model is used to reconstruct the predicted sea clutter signal $\tilde{c}[n+1]$. Here, the low-frequency component $\mathbf{s}[n]$ is also predicted by an RBF network for simulation simplicity. Note that $\mathbf{s}[n]$ can be modeled via different approaches, such as advanced intelligent methods or statistical models.

The output signal of the synthesis model is

$$
\tilde{c}[n+1]=\tilde{\mathbf{s}}[n+1]+\lambda \cdot \tilde{\mathbf{s}}[n+1] \odot \tilde{\mathbf{d}}[n+1]
$$

where $\tilde{\mathbf{s}}[n+1]$ and $\tilde{\mathbf{d}}[n+1]$ are the one-step predicted outputs of the RBF predictors A and B. $\lambda$ is an amplitude modulation coefficient, which is normally set as $\lambda=1$. When the target sea clutter series is spiky, $\lambda$ can be larger than 1 in order to get a reconstructed signal with stronger fluctuation. In this work, $\lambda$ is chosen manually to obtain a relatively good performance. Adaptive $\lambda$ setting algorithm

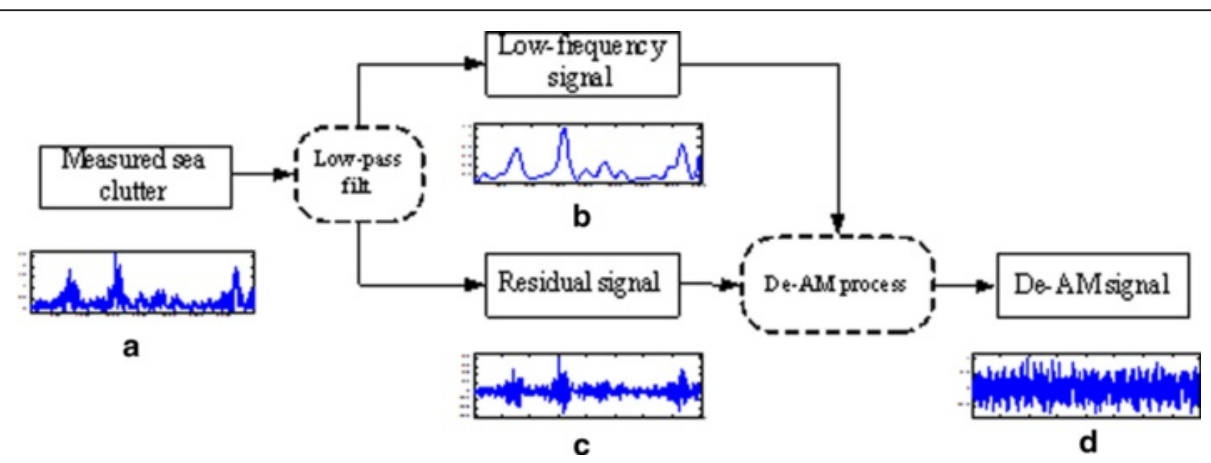

Fig. $\mathbf{6}$ a-d Decomposition process. This figure illustrates the process of decomposing the whole measured sea clutter into two components. The corresponding waveform examples are also presented 


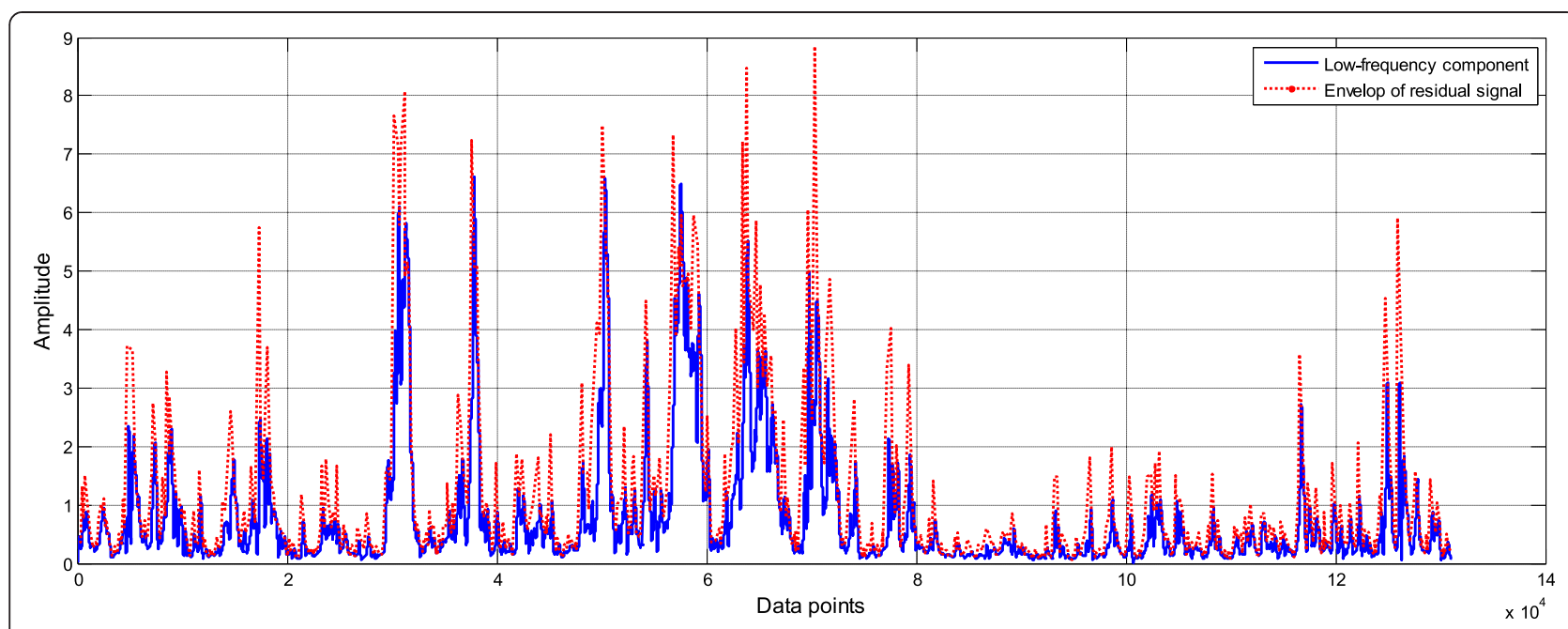

Fig. 7 Comparison of $s[n]$ and $\mathbf{r}[n]$. A comparison of two components after the first decompose step: low-frequency component $s[\mathbf{n}]$ and the envelope of residual signal $\mathbf{r}[n]$

should be considered in the future to improve the practicability of SCCS.

It should be mentioned that the measured sea clutter may include measurement noise and dynamic noise [1]. Since a determined chaotic process is noisefree, measurement noise and dynamic noise should be removed before approximating sea clutter time series with chaotic models. Unfortunately, due to the absence of a priori knowledge, these noise components can hardly be distinguished from measured sea clutter. So in this work, the contribution of noise components is assumed as a white noise floor in the frequency domain. In our simulation experiments, the input signal of the RBF predictor $\mathrm{B}$ is $\mathbf{d}[n]$ with the white noise floor removed.

\section{Simulation results \\ 4.1 Typical results}

(1) Partial chaotic data experiment

A partial chaotic series is simulated to validate the SCCS approach. The partial chaotic signal is constructed by a Lorenz chaotic signal and a low-frequency signal extracted from real data.
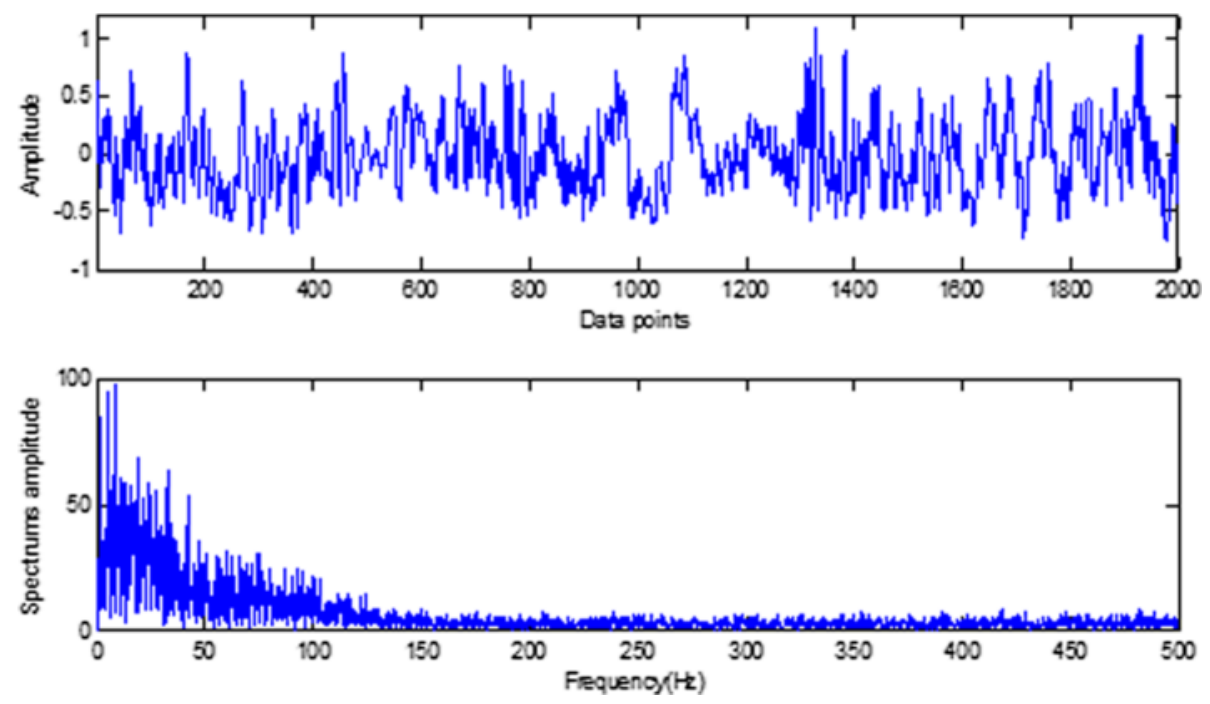

Fig. 8 Characteristics of the de-AM signal. The waveform and spectrum of a typical de-AM signal 


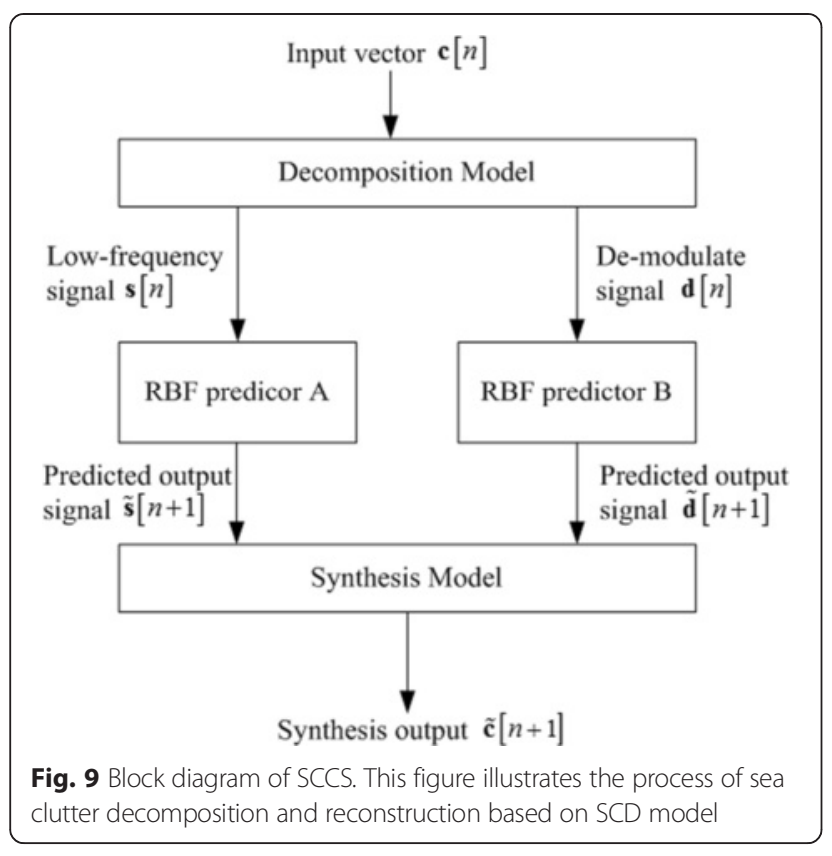

A typical waveform obtained via numerical integration of the Lorenz system described in Eq. (2) is illustrated in Fig. 10. The corresponding phase-space projection is shown in Fig. 11.

Here, signal $\mathbf{y}$ in the Lorenz system is utilized to construct the partial chaotic signal. Note that the amplitude of $\mathbf{y}$ is normalized to the amplitude range level of the real measured sea clutter series. Then the constituent partial chaotic series is

$$
\mathrm{s}_{p c}=\mathrm{s}+\mathrm{s} \odot \mathrm{y}
$$

where $\mathbf{s}$ represents the low-frequency signal in a sea clutter signal. In this work, $\mathbf{s}$ is a segment of the low-frequency signal extracted from data $\mathbf{S 4}$.

A typical waveform of the constituent partial chaotic signal $\mathrm{s}_{p c}$ is shown in the top subplot of Fig. 12. The low- frequency signal $\mathbf{s}$ is also illustrated for comparison, which can describe the fluctuant trend of $s_{p c}$. The normalized spectrum of $\mathrm{s}_{p c}$ is given in the bottom subplot of Fig. 12 . It can be observed that $s_{p c}$ has a relatively broad flat spectrum with a high-energy component in the very lowfrequency region, which is similar to the spectrum of real sea clutter shown in Fig. 5a. Note that this result is another proof of the decomposition model introduced in Section 3.2.

Then the proposed SCCS approach is applied to model the partial chaotic signal $s_{p c}$. Results are given in Fig. 13, where the vertical ordinate is the clutter signal amplitude. Five thousand points are used for training, and the next 2000 points are used for testing. Results show that the SCCS approach can excellently fit the constituent partial chaotic signal.

\section{(2) Real sea clutter data experiments}

In this part, the proposed SCCS approach is applied to model real sea clutter data introduced in Table 1. Three typical results using the dataset $S 4$ are depicted in Figs. 14, 15 , and 16, respectively. Five thousand points are used for training, and the next 2000 points are used for testing. The amplitude modulation coefficient $\lambda$ is fixed to 1.4 in the following experiments discussed in this work.

Results suggest that the SCCS approach can fit the spiky measured sea clutter without divergence phenomenon. Figure 14 shows that the proposed SCCS approach is able to fit measured data with large sea clutter spikes. In Fig. 15, the amplitude of some samples in the SCCS output are larger than that of the measured data. While in Fig. 16, the amplitude of some samples in the SCCS output are smaller than that of the measured data, and the approximation errors usually appear when the signal comes to wave crests. This kind of fitting error is the reason to involve the amplitude modulation coefficient

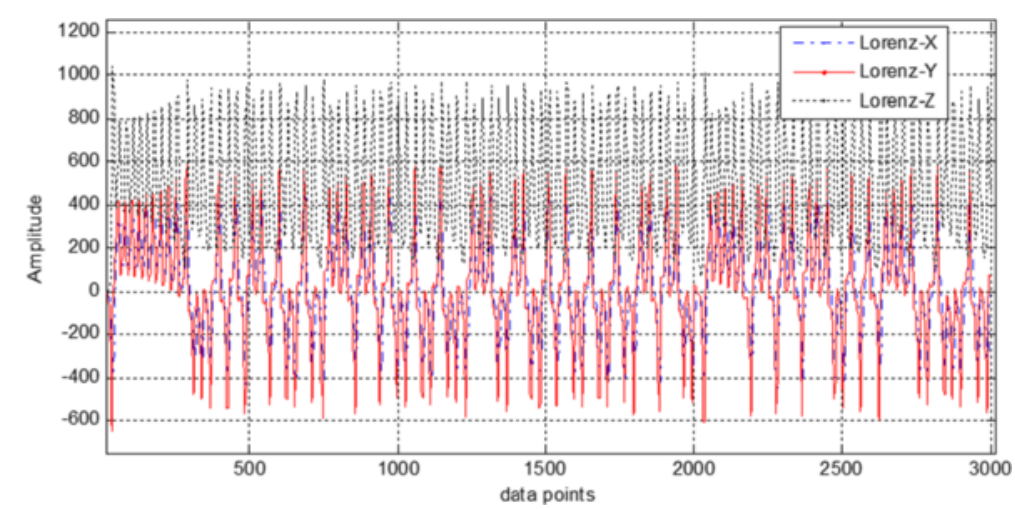

Fig. 10 A typical waveform obtained by Lorenz system. This figure illustrates a typical waveform obtained by a Lorenz system with parameters set as $\sigma=267, r=595$, and $b=100$ 


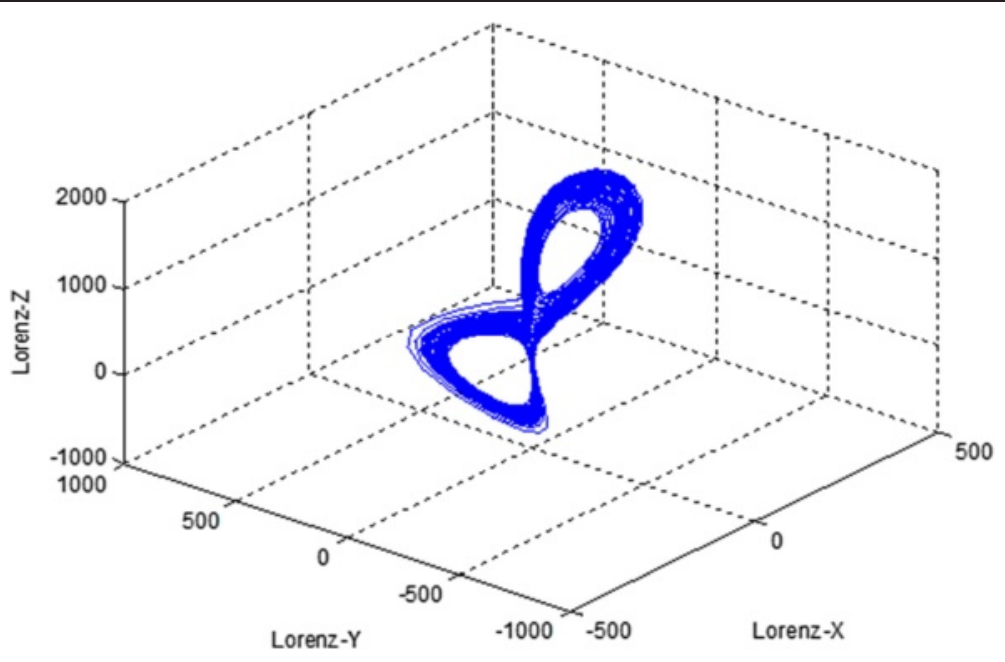

Fig. 11 Phase-space projection. A phase-space projection of the three signals shown in Fig. 10

$\lambda$ in Eq. (9), and these errors can be reduced by adjusting $\lambda$ to an appropriate value. Since $\lambda$ is fixed in the experiments discussed in this work, fitting results illustrated here are not optimal performance which the SCCS method can achieve.

\subsection{Performance comparison}

To further test the effectiveness of the SCCS approach, a longer series is reconstructed by SCCS. As mentioned earlier in Section 2, the predictable samples' number is also an important performance evaluation indicator in sea clutter modeling. In a longer series test, 5000 points are used in the training process and the next 6000 points are used for testing. In comparison, a same length series is predicted directly by a single-RBF network as described in Section 2, as well as an MM-RBF network with two single-RBF models and one gating model (2M-RBF).

In the 2M-RBF network utilized in this work, two RBF predictors (named as RBF-1 and RBF-2) are different in their spread coefficients. The spread coefficient is a variance parameter in the nonlinear transfer function in an RBF network and can be set as a multiple of the average distance between data points considered in the fitting process [18]. Therefore, by changing the spread coefficient, the network can be adjusted to fit different segments of sea clutter series with different average data distances. In this work, the spread coefficients in all single-RBF networks are set as $\sigma_{\mathrm{SRBF}}=1$. The spread coefficient of each RBF predictors in
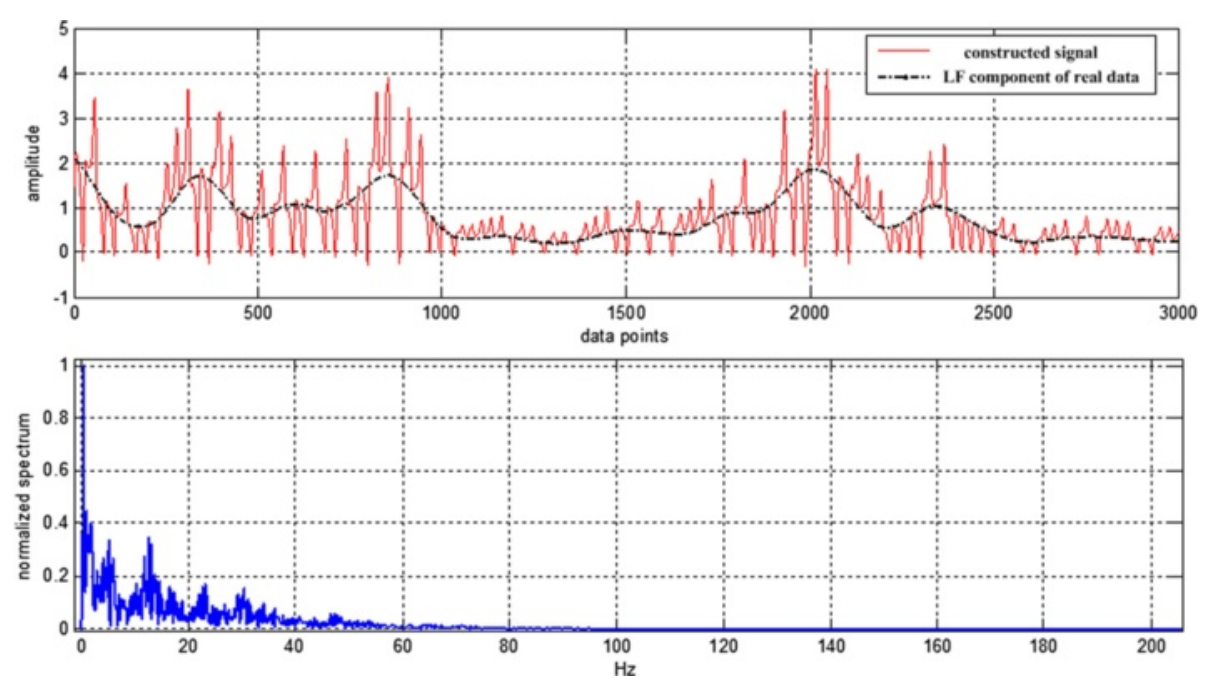

Fig. 12 Waveform and spectrums of the constituent partial chaotic signal. A typical waveform of the constructed partial chaotic signal and the low-frequency signal used to construct it are shown in the top subplot. The normalized spectrum of this typical partial chaotic signal is given in the bottom subplot 


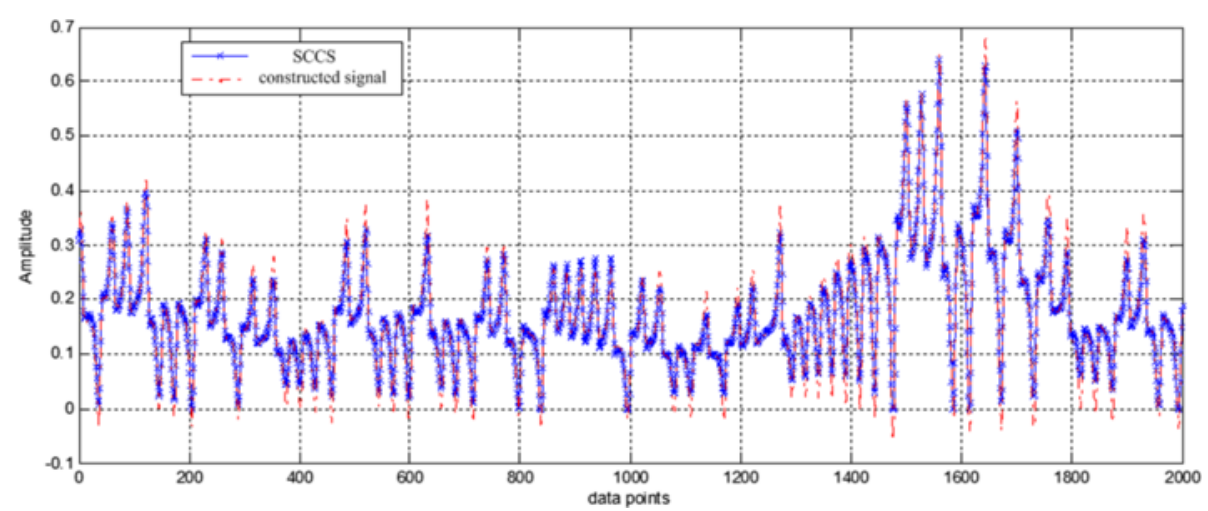

Fig. 13 SCCS prediction result with the partial chaotic signal. SCCS prediction result and the corresponding real data from the constructed partial chaotic signal

2M-RBF network is set as $\sigma_{\mathrm{MMRBF} 1}=1$ and $\sigma_{\mathrm{MMRBF} 2}=10$, respectively. Note that $\sigma_{\mathrm{MMRBF} 1}$ is set equal to $\sigma_{\mathrm{SRBF}}$ in order to give a comparative study.

In addition, in order to describe the time-decomposition modeling strategy of the MM-RBF network, the output of the gate model, i.e., the model choosing results are obtained from the posterior fitting accuracy. Note that in real applications, the output of the gating model should be trained with a priori information [9]. Let the prediction outputs of the two RBF predictors in 2M-RBF be $\tilde{\mathbf{c}}_{M 1}[n]$ and $\tilde{\mathbf{c}}_{M 2}[n]$, respectively. Output signal of the gate model in $2 \mathrm{M}-\mathrm{RBF}$ is given by:

$g[n+m]= \begin{cases}0, & \left|\tilde{c}_{M 1}[n+m]-c[n+m]\right| \leq\left|\tilde{c}_{M 2}[n+m]-c[n+m]\right| \\ 1, & \text { otherwise }\end{cases}$

Here $m=1,2, \ldots, 6000$. Then the final output of $2 \mathrm{M}$ RBF is

$$
\begin{aligned}
\tilde{c}_{M M}[n+m]= & (1-g[n+m]) \cdot \tilde{c}_{M 1}[n+m] \\
& +g[n+m] \cdot \tilde{c}_{M 2}[n+m]
\end{aligned}
$$

which means that in step $m$, if the gate model output is 0 , the RBF-1 predictor should be set active and the output of $2 \mathrm{M}-\mathrm{RBF}$ is equal to $\tilde{\mathbf{c}}_{M 1}[n]$; otherwise, the RBF-2 predictor should be set active and the output of $2 \mathrm{M}-\mathrm{RBF}$ is equal to $\tilde{\mathbf{c}}_{M 2}[n]$.

The fitting results are presented in Fig. 17. It shows that waveforms of the SCCS output fit the measured data well, while the predicted signal of the single-RBF approach becomes seriously divergent in some areas. As a sub-optimal performance fitting result, the output of $2 \mathrm{M}-\mathrm{RBF}$ is able to fit the real data in some segments, but incurs relatively large fitting errors in other areas.

Detail information of a typical data area without divergence or large fitting errors is presented in Fig. 18. In Fig. 18, waveforms as well as the gate model output $\mathbf{g}[n]$

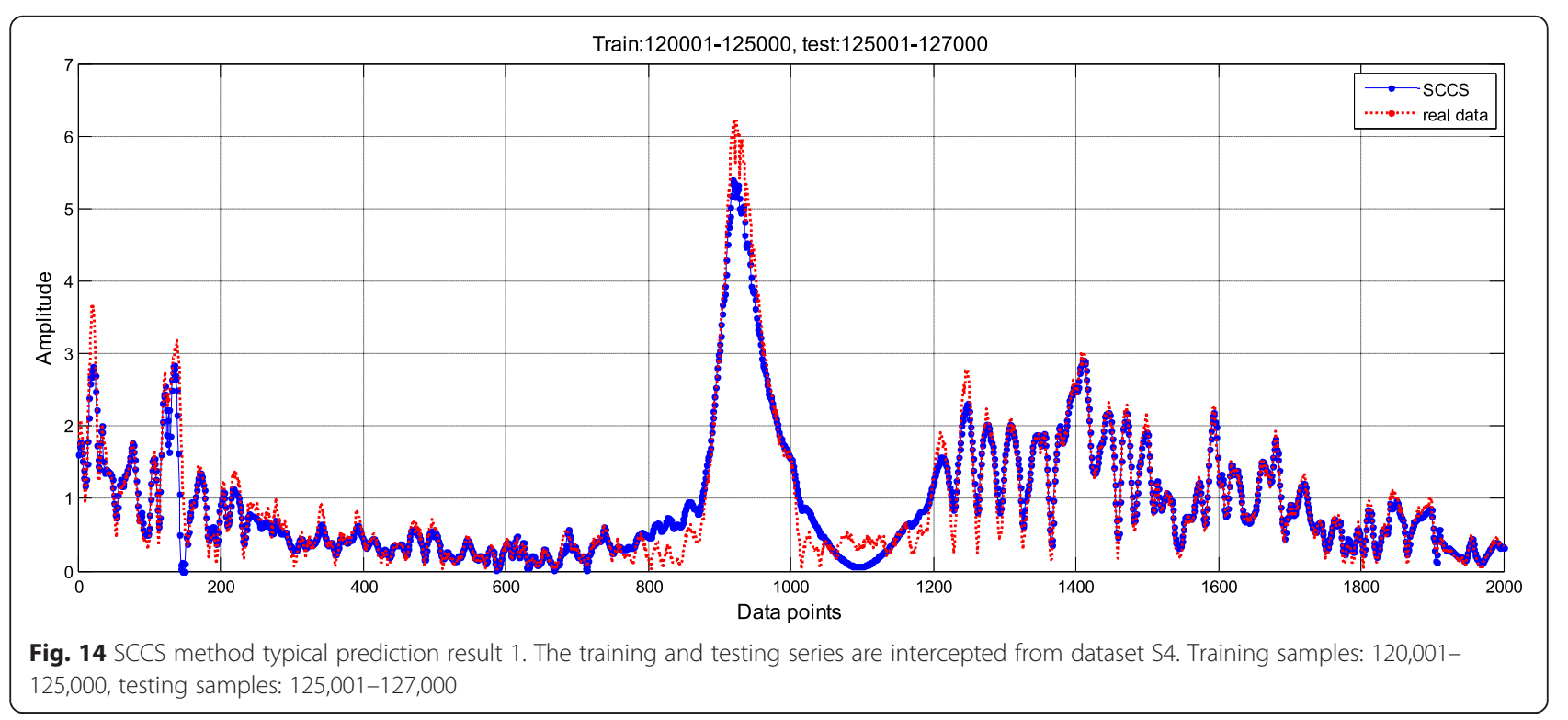




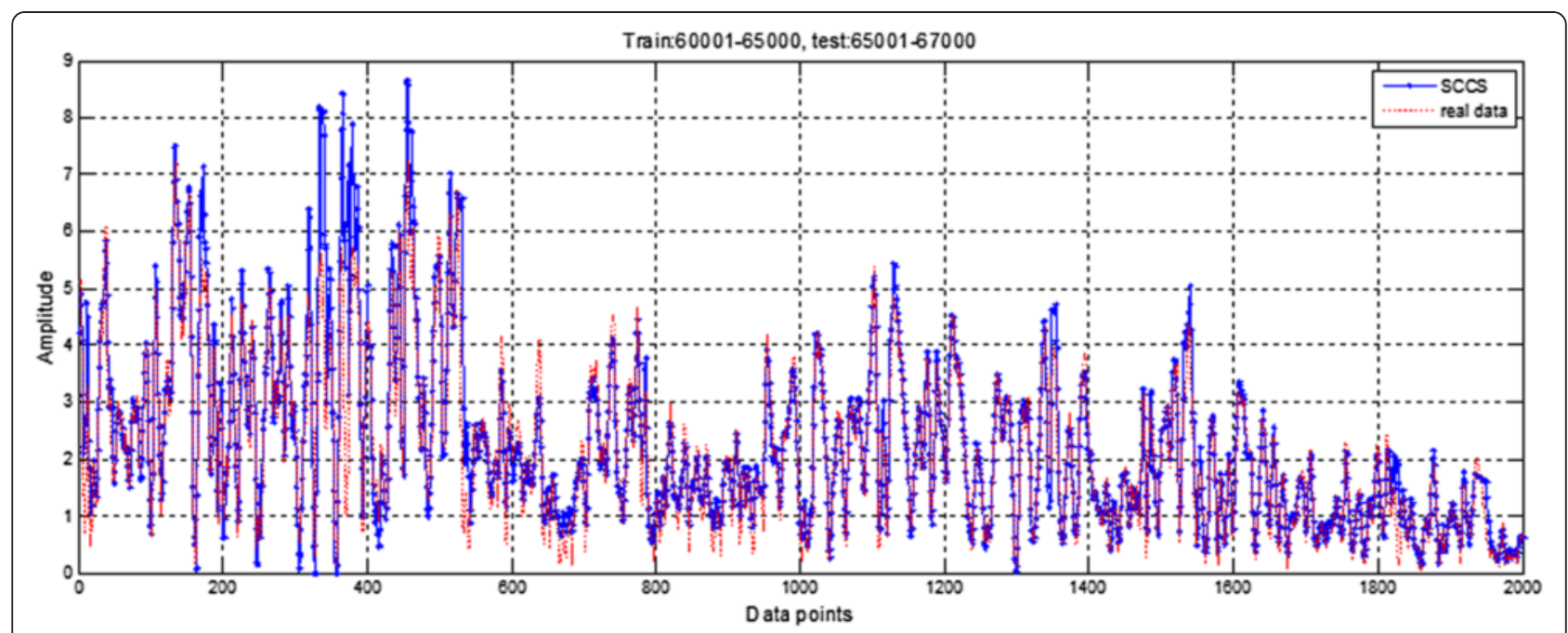

Fig. 15 SCCS method typical prediction result 2. The training and testing series are intercepted from dataset S4. Training samples: 60,001-65,000, testing samples: 65,001-67,000

from data point 475 to 520 are depicted. In the data point area from 475 to $485, \mathbf{g}[n]$ is stable at 1 , which means that in this data segment, RBF-2 in 2M-RBF has a better fitting performance and should be set as the active predictor. In this area, $2 \mathrm{M}-\mathrm{RBF}$ has a better fitting performance than single-RBF since RBF-2 is active. Meanwhile, in the data point area from 491 to $504, \mathbf{g}[n]$ is stable at 0 , which means that in this segment RBF-1 has a better performance. As shown in Fig. 18, in this segment the output of $2 \mathrm{M}-\mathrm{RBF}$ is identical to that of single-RBF since the spread coefficient of RBF-1 is equal to that of the single-RBF network. This result suggests that the RBF-2 model works worse than RBF-1 in this data segment, and none of the predictors in $2 \mathrm{M}-\mathrm{RBF}$ is suitable for this data segment. In contrast, the SCCS output fits the real data well in all the data segments, as shown in Fig. 17.
The comparison results show that the performance of single-RBF is unacceptable for its risk to diverge. The 2MRBF network is a better predictor than single-RBF, but still not stable since the number of predictors in MM-RBF is limited and cannot be very large in real applications. When none of them is suitable, the fitting performance will degrade. While for the proposed SCCS, since the sea clutter signal is decomposed before prediction, a good and stable fitting performance can be obtained by two simple-RBF networks without any parameter adjustment or gate model training process, which makes it a better choice for sea clutter modeling.

\subsection{Detection performance discussion}

A typical target detection scene is considered in this subsection to examine the detection performance of the

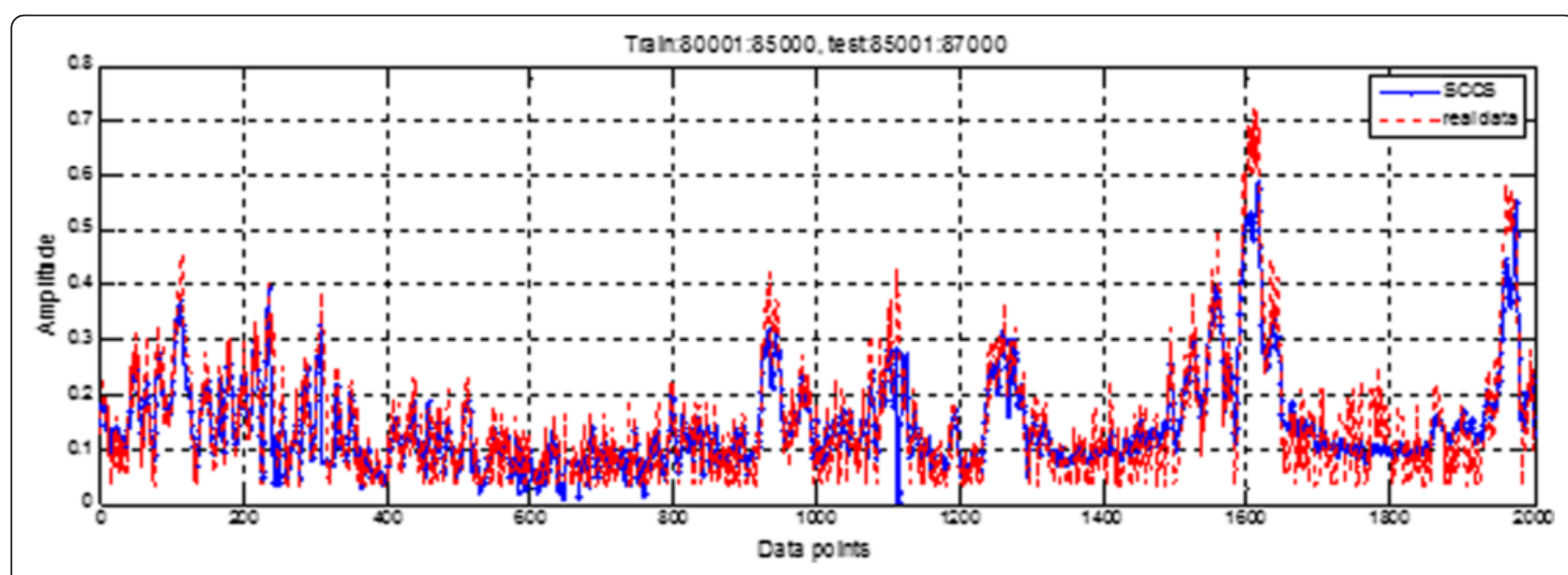

Fig. 16 SCCS method typical prediction result 3. The training and testing series are intercepted from dataset S4. Training samples: 80,001-85,000, testing samples: 85,001-87,000 


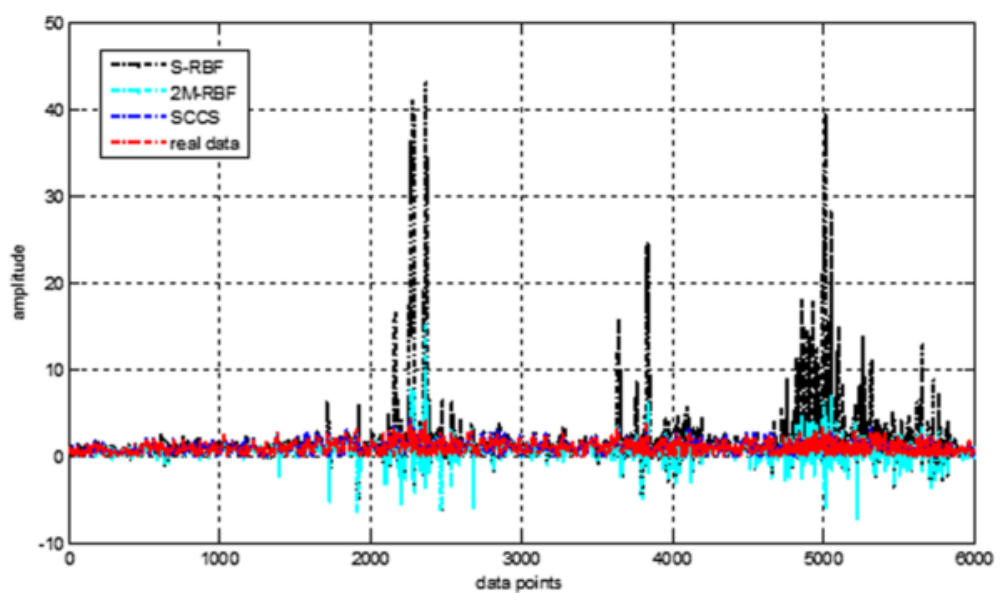

Fig. 17 Typical prediction results with longer testing series. Fitting performance comparison of single-RBF, MM-RBF predictor, and SCCS predictor. The measured data is intercepted from dataset \$4, 5000 samples are used for training and the next 6000 samples are used for testing

SCCS model. The target echo signal is assumed to exist in the $i$ th pulse, then the echo signal series is

$$
y[n]=\left\{\begin{array}{l}
s_{t}+c[n], n=i \\
c[n], \quad n \neq i
\end{array}\right.
$$

where $s_{t}=\sqrt{\mathrm{SCNR} \cdot P_{C N}} ; \mathrm{SCNR}$ refers to the signal to clutter-and-noise power ratio and $P_{C N}$ refers to the power of clutter and noise. Note that since the real sea clutter datasets include clutter echo and noise, $c[n]$ is actually the clutter-and-noise signal.

For the $n$th pulse, the output of the detector is

$$
d[n]= \begin{cases}1, & \text { if }|\tilde{c}[n]-y[n]| \geq \varepsilon \\ 0, & \text { otherwise }\end{cases}
$$

where $\tilde{c}[n]$ is the predicted output of SCCS and $\varepsilon$ is the detector threshold. The same 6000-point series shown in Fig. 17 is used for the detection experiment. Let $i$ changes from 1 to 6000 , then the detection probability is calculated as

$$
P_{d}=\sum_{i=1}^{6000} d[i] / 6000
$$

Figure 19 shows the $P_{d}$ results under different SCNR with three $\varepsilon$ values:

$$
\begin{aligned}
\varepsilon_{1} & =0.05 \cdot \operatorname{var}(\mathbf{c}[n]), \quad \varepsilon_{2}=0.1 \cdot \operatorname{var}(\mathbf{c}[n]), \quad \varepsilon_{3} \\
& =0.15 \cdot \operatorname{var}(\mathbf{c}[n])
\end{aligned}
$$

where $\operatorname{var}(\mathbf{c}[n])$ indicates the variance of $\mathbf{c}[n]$. In a low SCNR area (less than $-3 \mathrm{~dB}$ ) illustrated in Fig. 19, three curves are stable at values of $0.21,0.05$, and 0.01 separately. These values are equal to the false alarm probabilities under their $\varepsilon$ values. Since a false alarm will occur when the prediction error becomes larger than $\varepsilon$, for a determined threshold $\varepsilon$, the false alarm probability is proportional to

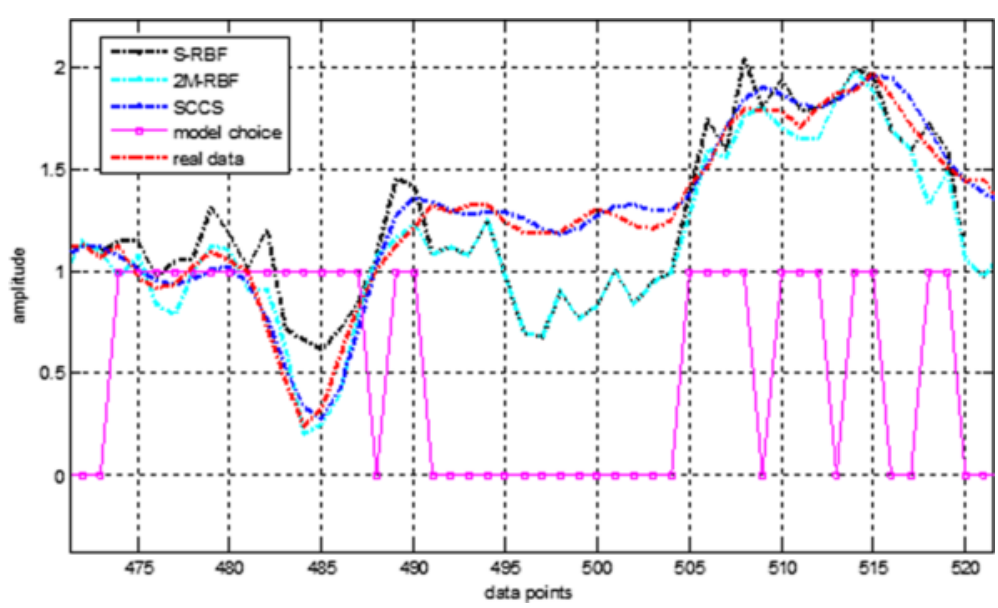

Fig. 18 Local maps of Fig. 17 with gate model output. A local map of Fig. 17 with waveforms from data point 475th to 520th as well as the output signal of gate model in $2 \mathrm{M}-\mathrm{RBF}$ predictor 


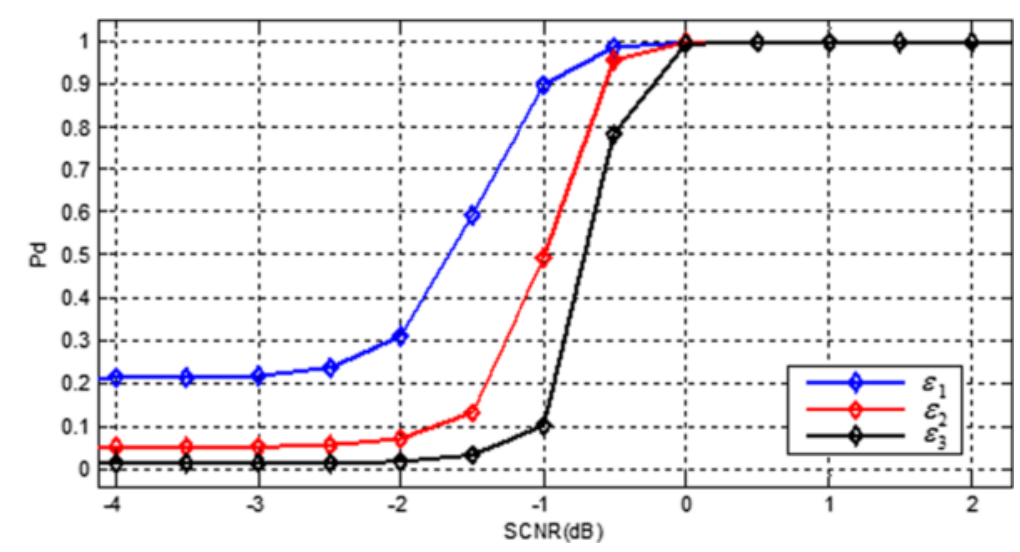

Fig. 19 Detection performances of SCCS with different threshold values. Detection performance when the target signal existing in a single pulse. The detection probability results obtained with different detector thresholds are illustrated

the prediction error level. On the other hand, for a determined prediction error level, the larger the threshold, the less the false alarm probability as well as the less the sensitivity of the detector. In real applications, if the threshold is set as $\varepsilon_{3}$, the false alarm probability is 0.01 , and the existing target in a single pulse can be detected if its power is comparable to the clutter-and-noise power.

Note that under this detection scenario, a target signal in a single pulse can hardly be detected from the waveform or spectrum, especially when the target is near or overlapped by the sea spikes.

\subsection{Applicability tests}

To further evaluate the applicability of the SCCS approach in different situations, we consider applying SCCS to fit the five real measured datasets introduced in Table 1. The root-mean-squared error (RMSE) is used as the performance measurement:

$$
\operatorname{RMSE}_{d, l}=\frac{\sqrt{\sum_{m=1}^{M}(\tilde{\mathbf{c}}[n+m]-\mathbf{c}[n+m])^{2}}}{M}
$$

where $M$ refers to the number of testing samples, $d$ refers to the dataset number, and $l$ refers to the data start point in each dataset. Five thousand samples are used for training, and the next 2000 samples are used for testing $(M=2000)$.

To get a fair and comprehensive performance evaluation, seven series from each dataset are used for training and testing. The start points of the seven series in each dataset are 1, 20,000, 40,000, 60,000, 80,000, 100,000, and 120,000, respectively. So we have $d=[1,2,3,4,5], l=[1,20,000,40$, 000, 60, 000, 80, 000, 100, 000, 120,000]. The RMSE of 35 tests (5 datasets, 7 series in each dataset) are shown in Table 2.

Results show that for all the 35 tests, the fitting RMSE remains under 0.1, which means that the SCCS approach is able to reconstruct measured sea clutter series under different sea state situations.

To evaluate the relationship between the fitting performance and sea clutter characteristics, mean value $\varepsilon_{d}$ and variance $\sigma_{d}$ of the fitting RMSE of each dataset are calculated as:

$$
\begin{aligned}
\varepsilon_{d} & =\sum_{l} \operatorname{RMSE}_{d, l} / 7 \\
\sigma_{d} & =\sum_{l}\left(\operatorname{RMSE}_{d, l}-\varepsilon_{d}\right)^{2} / 7
\end{aligned}
$$

Results of $\varepsilon_{1} \sim \varepsilon_{5}$ and $\sigma_{1} \sim \sigma_{5}$ are shown in Fig. 20, where

\begin{tabular}{|c|c|c|c|c|c|c|c|c|}
\hline Datasets & Wave heights (m) & Series 1 RMSE & Series 2 RMSE & Series 3 RMSE & Series 4 RMSE & Series 5 RMSE & Series 6 RMSE & Series 7 RMSE \\
\hline S2(Lo-State) & 0.8 & 0.0075 & 0.0029 & 0.0067 & 0.0039 & 0.0034 & 0.0216 & 0.0357 \\
\hline S4(\#31) & 0.9 & 0.0089 & 0.0009 & 0.0005 & 0.0226 & 0.0002 & 0.0019 & 0.0041 \\
\hline S5(\#280) & 1.4 & 0.0057 & 0.0005 & 0.0283 & 0.0005 & 0.0010 & 0.0006 & 0.0011 \\
\hline S1(Hi-State) & 1.8 & 0.0102 & 0.0137 & 0.0102 & 0.0429 & 0.0676 & 0.0085 & 0.0037 \\
\hline S3(\#19) & 2.0 & 0.0095 & 0.0732 & 0.0085 & 0.0068 & 0.0071 & 0.0082 & 0.0079 \\
\hline
\end{tabular}
the $x$ axis is the wave height of each dataset. As mentioned

Table 2 RMSE of 35 tests 


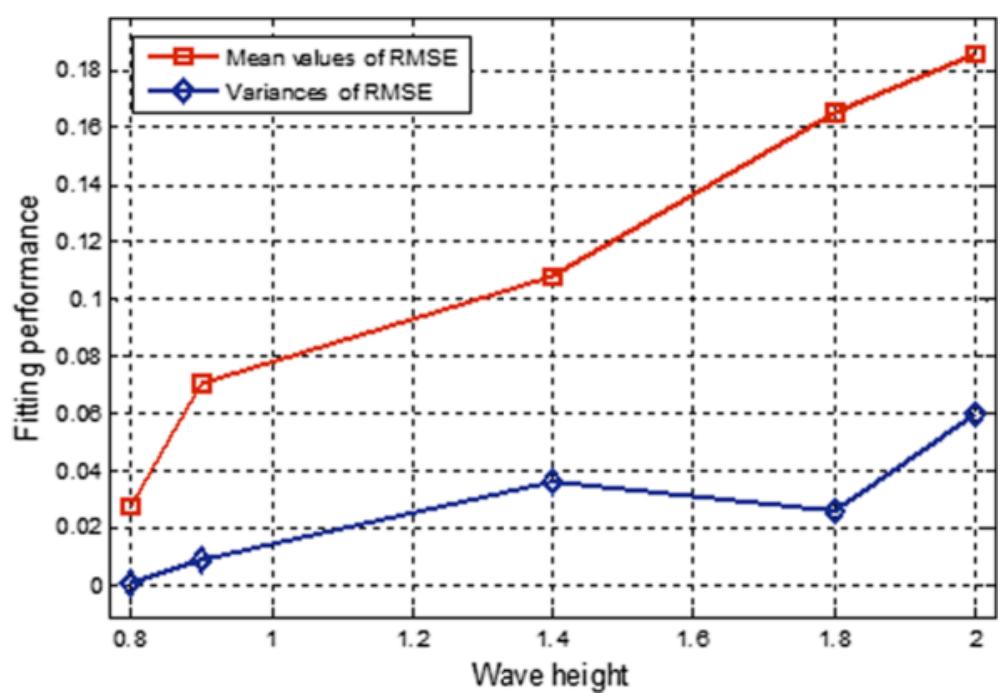

Fig. 20 Mean values and variance values of RMSE results with measured data collected under different wave height. The RMSE results are calculated with Eq. (17). The mean values and variance values of RMSE results are calculated with Eq. (18)

above, the wave height can be used as a measure of sea state.

It can be observed that as the wave height increases, the mean values of RMSE are monotonously increasing, and the variances have a certain upward trend. This indicates that the fitting performance of SCCS has a kind of proportional relationship with the sea state. The reason might lie in the fact that under a high sea state circumstance, the increased degree of freedom and fluctuation in sea clutter makes it more difficult to approximate the de-AM signal with a structure fixed neural network. However, comparing to the prediction outputs of the single-RBF method, the SCCS outputs remain convergent in all the experiments. Improved neural networks can be used to deal with this sea state related performance deterioration.

Based on the real data involved experiment results, the following observations could be made about the SCCS modeling approach.

(1) Free of divergence problem The SCCS approach can fit sea clutter with different waveform properties and barely encounter divergence problem, which obviously outperforms the single-RBF approach since the divergence problem can cause unacceptable prediction errors.

(2) Stable performance when long testing series is needed

Keeping the training samples' number unchanged, with the increase of the testing samples' number, the SCCS approach consistently maintains a stable fitting performance. This indicates that the trained SCCS model successfully approximates the compound underlying process within the target sea clutter.

(3) Applicable under different sea states Sea clutter collected in various sea states can be quite different. For most of the clutter datasets, we have investigated, the SCCS approach has shown a good overall fitting performance.

These observations confirm the effectiveness of the SCCS approach. Most of them come from the utilization of the SCD model proposed in this paper, in which a more suitable component for chaotic modeling is found. Again, we emphasize that the role of the SCD model is a preprocessing approach, which can be used to reduce the difficulty of sea clutter modeling. So the SCD-SCCS approach is not exclusive. RBF predictors in SCCS can be replaced by other modeling approach (such as improved intelligent algorithms, fractal models, and statistics models) as an alternative choice in different application situations.

\section{Conclusions}

In this paper, a sea clutter decomposition model is proposed inspired by the observation that sea clutter as a whole signal is not similar enough to chaotic signals. Based on the comparison analysis, a frequency domain divided decomposition model is proposed, in which sea clutter signals can be decomposed into a slow-frequency component and a de-modulated component. Based on the decomposition model, a sea clutter constituent synthesis approach is developed to reconstruct sea clutter.

Supported by experiments using actual measured sea clutter signals, the SCCS approach is found to outperform the single-RBF approach. It is also demonstrated 
that the SCCS approach can fit sea clutter effectively in different sea states. Future work may focus on the adaptive adjustment algorithm of the amplitude modulation coefficient in the SCCS model.

\section{Competing interests}

The authors declare that they have no competing interests.

\section{Acknowledgements}

This work is supported by National Natural Science Foundation of China under Contract (NSFC 61201280 NSFC 61371184 NSFC 61401062).

Received: 23 January 2015 Accepted: 17 August 2015

Published online: 26 August 2015

\section{References}

1. S Haykin, "Adaptive Radar Signal Processing", A John Wiley \& Sons, Inc., Publication, 2007.

2. G Davidson, Simulation of coherent sea clutter". IET Radar Sonar Navig 4(2), 168-177 (2010)

3. E Conte, M Longo, M Lops, "Modeling and simulation of non-Rayleigh radar clutter," IEE Proceedings-F, 1991,pp. 121-130,

4. AF Fay, J Clarke, SR Peters, "Weibull distribution applied to sea-clutter", Proc. IEE Conf. Radar'77, London, UK, 1977, pp. 101-103.

5. HC Chan, Radar sea-clutter at low grazing angles. Proc. Inst. Elect. Eng. F137, 102-112 (1990)

6. CJ Baker, "K-distributed coherent sea clutter," IEE Proceedings-F, 1991, pp. 89-92.

7. S Watts, "Radar detection prediction in K-distributed sea clutter and therma noise". IEEE Transaction on aerospace and electronic systems AES-23(1), 40-45 (1987)

8. F Gini, Performance analysis of two structured covariance matrix estimators in compound-Gaussian clutter. Signal Process. 80, 365-371 (2000)

9. N Xie, H Leung, $\mathrm{H}$ Chan, "A multiple_model prediction approach for sea clutter model". IEEE Trans Geosci Remote Sens 41(6), 1491-1502 (2003)

10. CP Unsworth, MR Cowper, S McLaughlin, B Mulgrew, "False Detection of Chaotic Behavior in the Stochastic Compound K-Distribution Model of Radar Sea Clutter," Statistical Signal and Array Processing, Proceedings of the Tenth IEEE Workshop, 2000pp: 296-300,.

11. PR Chakravarthi, "Radar target detection in chaotic clutter," Radar Conference, IEEE National ,1997,pp: 367 - 371

12. J Hu, JB Gao, LF Posner, Y Zheng, WW Tung, "Target detection within sea clutter: A comparative study by fractal scaling analyses". Fractals 14(3), 187-204 (2006)

13. XK Xu, "Low observable targets detection by joint fractal properties of sea clutter: An experimental study of IPIX OHGR datasets". IEEE Trans Antennas Propag 58, 4 (2010)

14. J Guan, NB Liu, Y Huang, Y He, "Fractal characteristic in frequency domain for target detection within sea clutter". IET Radar Sonar Navig 6(5), 293-306 (2012)

15. S Haykin, $\mathrm{H}$ Leung, "Chaotic modeling of radar backscatter from an ocean surface," Ocean Technologies and Opportunities in the Pacific for the 90's Proceedings, 1991, pp: 1713 - 1716

16. N He, S Haykin, "Chaotic modeling of sea clutter". Electron Lett 28(28), 2076-2077 (1992)

17. S Haykin, S Puthusserypady, "Chaos, sea clutter, and neural networks". Signals Systems Comput Conf 2, 1224-1227 (1997)

18. MK McDonald, V Varadan, H Leung, "Chaotic Behaviour and Non-Linear Prediction of Airborne Radar Sea Clutter Data," Radar Conference 2002 Proceedings of the IEEE, 2002, pp:331 - 337.

19. MR Cowper, B Mulgrew, CP Unsworth, "Nonlinear prediction of chaotic signals using a normalized radial basis function network, " Signal Processing, 2002, pp:775 - 789 .

20. N Xie, H Leung, "Reconstruction of piecewise chaotic dynamic using a Genetic Algorithm multiple model approach". IEEE Trans Circuits Syst I: Regul Pap 51(6), 1210-1222 (2004)

21. J Park, IW Sandberg, "Universal approximation using radial-basis-function networks". Neural comput 3(2), 246-257 (1991)

22. DS Broomhead, D Lowe, "Radial basis functions, multi-variable functional interpolation and adaptive networks". Royal Signals and Radar Establishment Malvern ( United Kingdom), 1988.
23. MJD Powell, "Radial basis functions for multivariable interpolation: a review", Algorithms for approximation. Clarendon Press, 1987: 143-167.

24. B Whitehead, TD Choate, "Cooperative-competitive genetic evolution of radial basis function centers and widths for time series prediction". Neural Netw IEEE Trans 7(4), 869-880 (1996)

25. RJ Schilling, JJ Carroll Jr, AF Al-Ajlouni, "Approximation of nonlinear systems with radial basis function neural networks". Neural Netw. IEEE Trans 12(1), $1-15$ (2001)

26. S Haykin, R Bakker, BW Currie, "Uncovering nonlinear dynamics-the case study of sea clutter". Proc. IEEE 90(5), 860-881 (2002)

27. H Leung, T Lo, S Wang, "Prediction of noisy chaotic time series using an optimal radial basis function neural network". Neural Netw. IEEE Trans 12(5), 1163-1172 (2001)

28. H Leung, T Lo, "Chaotic radar signal processing over the sea". IEEE J. Ocean. Eng. 18(3), 287-295 (1993)

29. HongYan X, ZhengDong Q, Wei X., "Weak signal estimation in chaotic clutter using selective support vector machine ensemble". 2012.

30. MS Willsey, KM Cuomo, AV Oppenheim, "Quasi-Orthogonal Wideband Radar Waveforms Based on Chaotic Systems". IEEE trans Aerosp Electron Syst 47, 3 (2011)

\section{Submit your manuscript to a SpringerOpen ${ }^{\odot}$ journal and benefit from:}

- Convenient online submission

Rigorous peer review

- Immediate publication on acceptance

- Open access: articles freely available online

- High visibility within the field

- Retaining the copyright to your article

Submit your next manuscript at $>$ springeropen.com 